\title{
Supporting Information \\ Novel reversible-binding PET ligands for imaging monoacylglycerol lipase based on piperazinyl azetidine scaffold
}

Jian Rong ${ }^{\mathrm{a}, \uparrow}$, Wakana Morib ${ }^{\mathrm{b}, \dagger}$, Xiaotian Xia ${ }^{\mathrm{a}}$, Michael A. Schafroth, ${ }^{\mathrm{c}}$ Chunyu Zhao ${ }^{\mathrm{a}}$, Richard S.

Van ${ }^{\mathrm{d}}$, Tomoteru Yamasaki, ${ }^{\mathrm{b}}$ Jiahui Chen ${ }^{\mathrm{a}}$, Zhiwei Xiao ${ }^{\mathrm{a}}$, Ahmed Haidera ${ }^{\mathrm{a}}$ Daisuke Ogasawara ${ }^{\mathrm{c}}$, Atsuto Hiraishi ${ }^{\mathrm{b}}$, Tuo Shao ${ }^{\mathrm{a}}$, Yiding Zhang ${ }^{\mathrm{b}}$, Zhen Chen, ${ }^{a}$ Fuwen Pang ${ }^{\mathrm{a}}$, Kuan $\mathrm{Hu}^{\mathrm{b}}$, Lin Xie, Masayuki Fujinaga ${ }^{\mathrm{b}}$, Katsushi Kumata, ${ }^{\mathrm{b}}$ Yuancheng Gou, ${ }^{\mathrm{e}}$ Yang Fang, ${ }^{\mathrm{e}}$ Shuyin Gu, ${ }^{\mathrm{e}}$ Huiyi Wei, ${ }^{\mathrm{f}}$ Liang Bao, ${ }^{\mathrm{e}} \mathrm{Hao} \mathrm{Xu},{ }^{\mathrm{f}}$ Thomas L. Collier ${ }^{\mathrm{a}}$, Yihan Shao ${ }^{\mathrm{d}}$, Richard E. Carsong, Benjamin F. Cravatt ${ }^{\mathrm{c}}$, Lu Wang ${ }^{\mathrm{f}, *}$, Ming-Rong Zhang,*, Steven H. Liang ${ }^{\mathrm{a}, *}$

${ }^{\mathrm{a} D i v i s i o n}$ of Nuclear Medicine and Molecular Imaging, Massachusetts General Hospital \& Department of Radiology, Harvard Medical School, Boston, MA 02114, USA

${ }^{\mathrm{b}}$ Department of Advanced Nuclear Medicine Sciences, National Institute of Radiological Sciences, National Institutes for Quantum and Radiological Science and Technology, Chiba 263-8555, Japan 'The Skaggs Institute for Chemical Biology and Department of Chemical Physiology, The Scripps Research Institute, SR107 10550 North Torrey Pines Road, La Jolla, CA 92037, USA

${ }^{\mathrm{d}}$ Department of Chemistry and Biochemistry, University of Oklahoma, Norman, OK 73019, USA ${ }^{\mathrm{e} C h e m s h u t t l e ~ I n c o r p o r a t i o n, ~} 1699$ Huishan Blvd., Wuxi, Jiangsu 214174, China

${ }^{\mathrm{f}}$ Center of Cyclotron and PET Radiopharmaceuticals, Department of Nuclear Medicine and PET/CT-MRI Center, The First Affiliated Hospital of Jinan University, Guangzhou 510630, China ${ }^{g}$ Department of Radiology and Biomedical Imaging, Yale University School of Medicine, New Haven, CT 06520, USA

*Corresponding authors. E-mail addresses: 1_wang1009@jnu.edu.cn (Lu Wang); zhang.mingrong@qst.go.jp (Ming-Rong Zhang); liang.steven@mgh.harvard.edu (Steven H. Liang). 
Table of contents

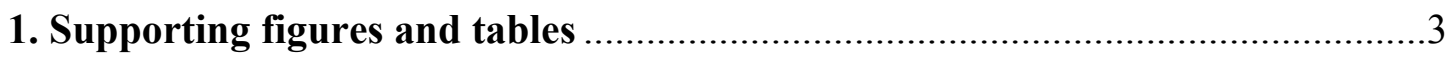

Figure S1 Off-target pharmacological evaluation of compound 15........................

Figure S2 Chase studies of $\left[{ }^{18} \mathrm{~F}\right] 10$ and $\left[{ }^{18} \mathrm{~F}\right] 15$ with JNJ-42226314 in mice ...........3

Figure S3 Representative time-activity curves (TACs) of $\left[{ }^{18} \mathrm{~F}\right] \mathbf{1 0}$ and $\left[{ }^{18} \mathrm{~F}\right] \mathbf{1 5}$ in whole monkey brain PET imaging studies................................................................ 4

Figure S4 Brain-to-blood ratio with metabolite-correction of $\left[{ }^{18} \mathrm{~F}\right] 10$ and $\left[{ }^{18} \mathrm{~F}\right] 15$ in

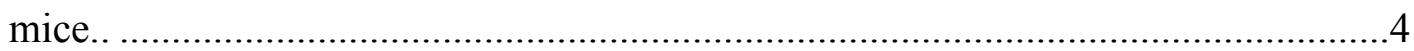

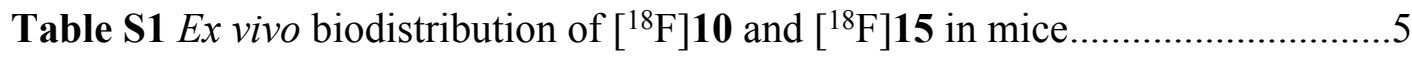

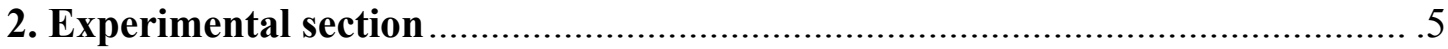

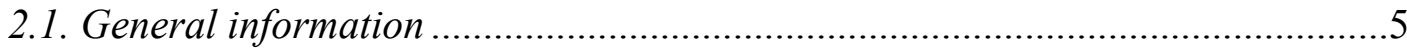

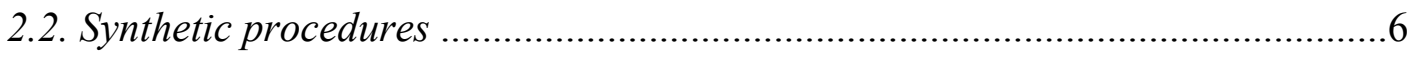

2.3. HPLC traces of key compounds ................................................................6 


\section{Supporting figures and tables}

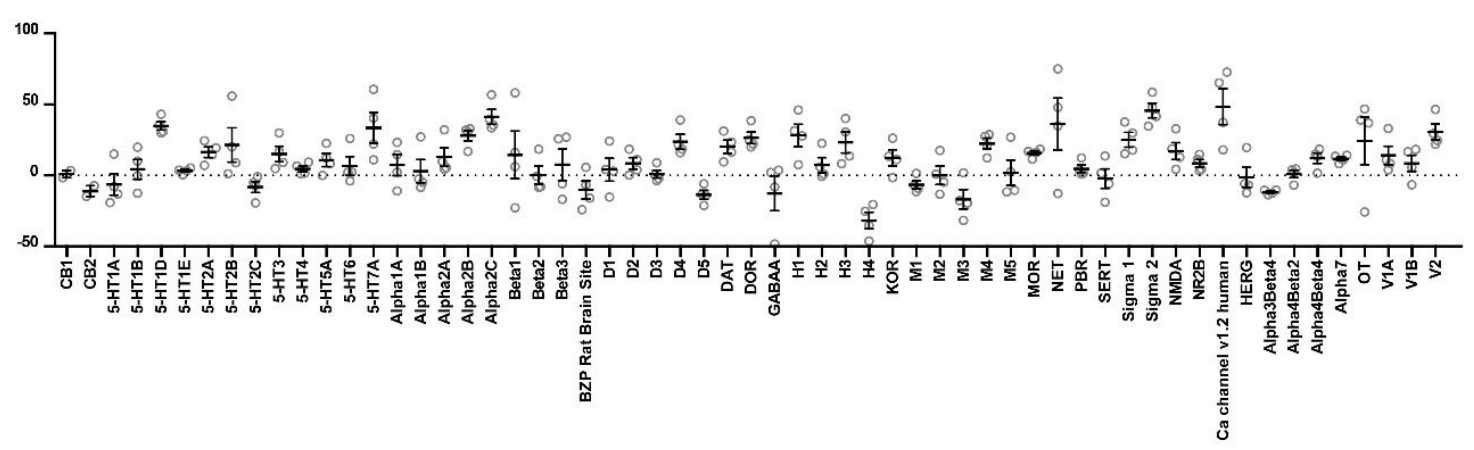

Figure S1. Off-target pharmacological evaluation of compound $\mathbf{1 5}$ at a concentration of $10 \mu \mathrm{M}$ against major CNS targets, including common GPCRs, enzymes, ion channels and transporters: Initial screening at a concentration of $10 \mu \mathrm{M}$. All data are mean \pm SEM (n $\geqslant 2)$. No significant off-target binding $(>50 \%)$ was observed at $10 \mu \mathrm{M}$ compound testing concentration.

A

A. Chase studies of $\left[{ }^{18} \mathrm{~F}\right] 10$ with JNJ-42226314

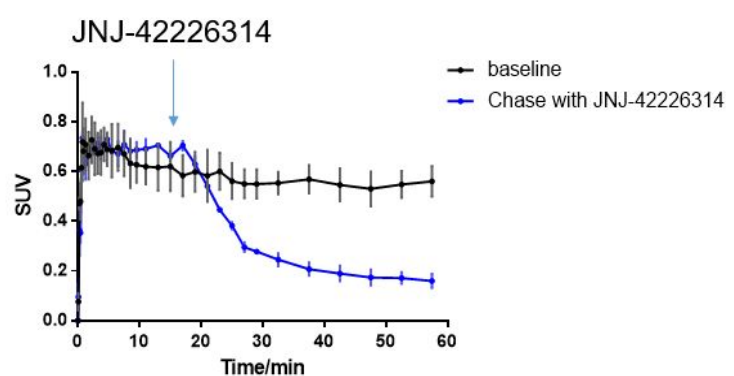

B

B. Chase studies of $\left[{ }^{18} \mathrm{~F}\right] 15$ with JNJ-42226314

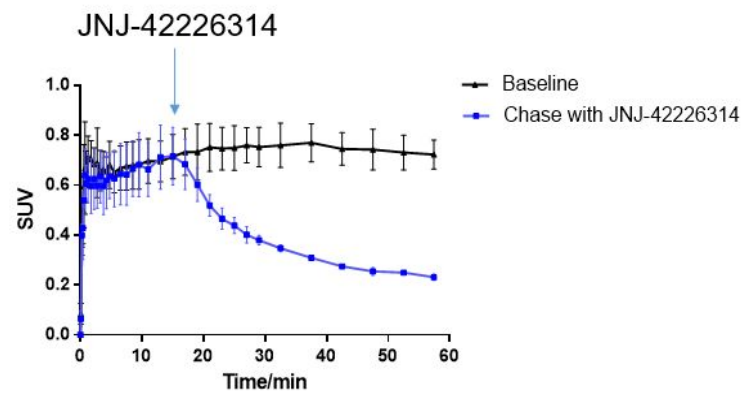

Figure S2. Chase studies of $\left[{ }^{18} \mathrm{~F}\right] 10$ and $\left[{ }^{18} \mathrm{~F}\right] 15$ with $\mathrm{JNJ}-42226314(3 \mathrm{mg} / \mathrm{kg})$ at $15 \mathrm{~min}$ post tracer administration in mice. All data are mean $\pm \mathrm{SD}(\mathrm{n} \geqslant 2)$. 
A

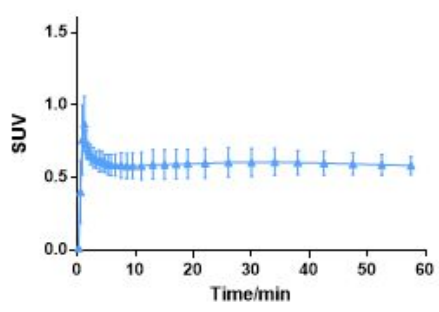

B

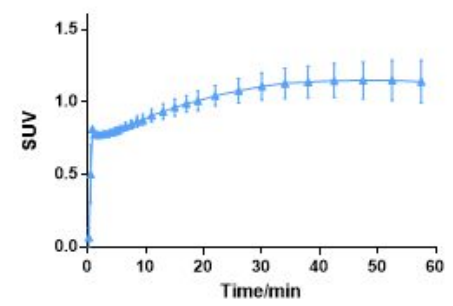

C

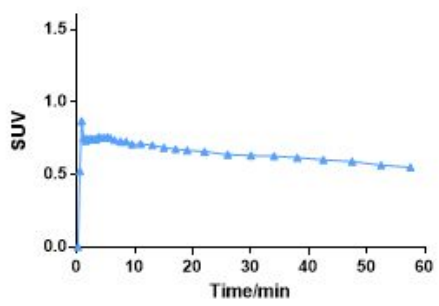

Figure S3. (A) Representative time-activity curve (TAC) of $\left[{ }^{18} \mathrm{~F}\right] \mathbf{1 0}$ in whole monkey brain under baseline conditions; (B) Representative time-activity curve (TAC) of $\left[{ }^{18} \mathrm{~F}\right] \mathbf{1 5}$ in whole monkey brain under baseline conditions; (C) Representative time-activity curve (TAC) of $\left[{ }^{18} \mathrm{~F}\right] 15$ in whole monkey brain with blocking reagent JNJ-42226314 (0.9 mg/kg). All data are mean $\pm \mathrm{SD}(\mathrm{n} \geqslant 2)$.

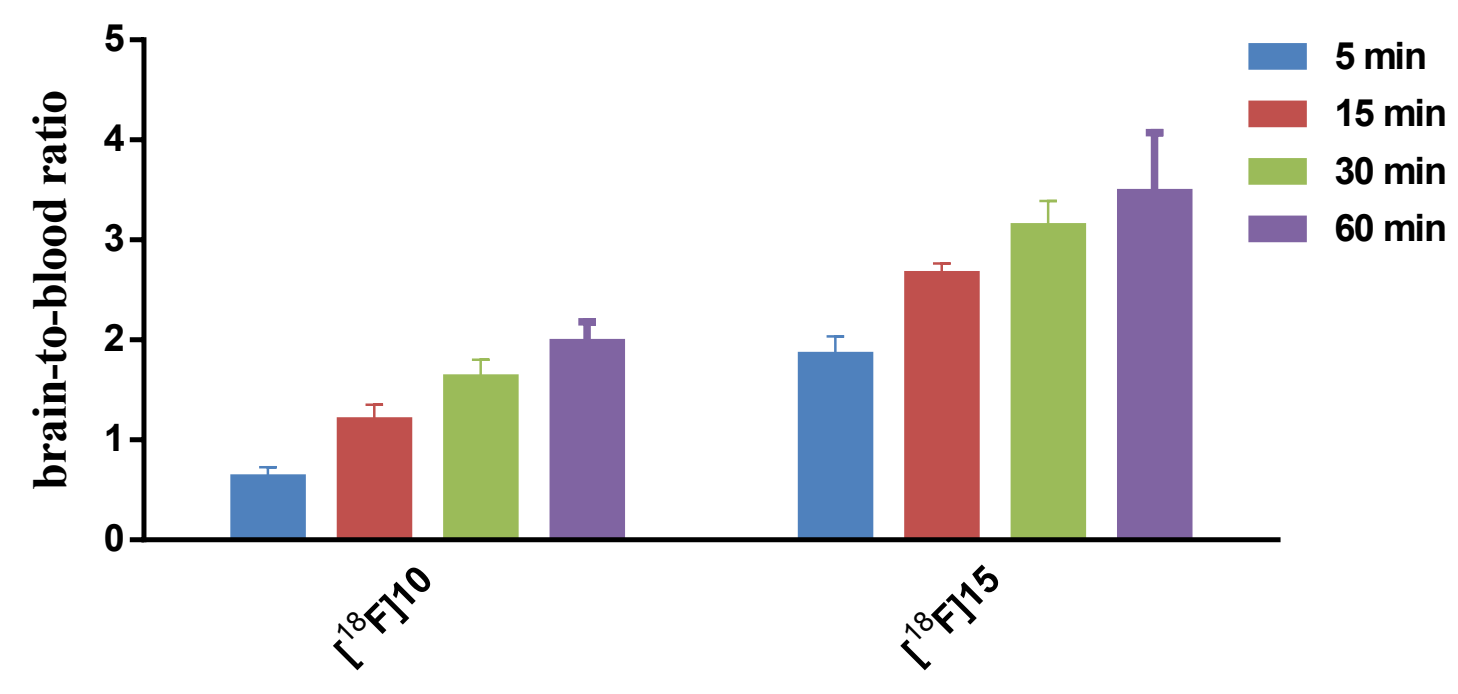

Figure S4. Brain-to-blood ratio with metabolite-correction of $\left[{ }^{18} \mathrm{~F}\right] 10$ and $\left[{ }^{18} \mathrm{~F}\right] 15$ in mice. All data are mean $\pm \mathrm{SD}(\mathrm{n}=2)$. 
Table S1 Ex vivo biodistribution of $\left[{ }^{18} \mathrm{~F}\right] 10$ and $\left[{ }^{18} \mathrm{~F}\right] 15$ in mice. Data are expressed as $\% \mathrm{ID} / \mathrm{g}($ mean $\pm \mathrm{SD}, n=4)$.

\begin{tabular}{|c|c|c|c|c|c|c|c|c|}
\hline \multirow[t]{2}{*}[{}^{18}\mathrm{F}]{$10 \quad(\mathrm{JNJ}-42226314)$} & \multicolumn{2}{|c|}{$5 \mathrm{~min}$} & \multicolumn{2}{|c|}{15 min } & \multicolumn{2}{|c|}{$30 \mathrm{~min}$} & \multicolumn{2}{|c|}{$60 \mathrm{~min}$} \\
\hline & Mean & $\mathrm{SD}$ & Mean & $\mathrm{SD}$ & Mean & $\mathrm{SD}$ & Mean & $\mathrm{SD}$ \\
\hline Brain & 2. 14 & 0.16 & 2.08 & 0.22 & 2.43 & 0.16 & 2. 39 & 0.16 \\
\hline Blood & 3. 02 & 0.26 & 1.53 & 0.17 & 1.32 & 0.20 & 1.07 & 0.10 \\
\hline Muscle & 4.65 & 1.04 & 3.92 & 0.56 & 3.73 & 0.34 & 2. 96 & 0.99 \\
\hline Spleen & 5.89 & 1.21 & 5.31 & 0.95 & 5.32 & 0.32 & 4.87 & 0.89 \\
\hline Heart & 31.36 & 4.26 & 20.03 & 4. 46 & 13. 12 & 1.98 & 8.81 & 0.94 \\
\hline Lung & 19.35 & 3.86 & 8.11 & 0.85 & 6.29 & 1.35 & 5.60 & 1.05 \\
\hline Pancreas & 9. 51 & 0.70 & 6.96 & 1.23 & 6.05 & 0.98 & 4.68 & 0.40 \\
\hline Stomach & 2.86 & 0.80 & 2.87 & 0.86 & 2.77 & 0.18 & 3.46 & 0.32 \\
\hline Small Intestine & 8.12 & 0.19 & 7.29 & 1.53 & 7. 49 & 1.09 & 8.60 & 2.83 \\
\hline Kidney & 22.22 & 3.29 & 15.59 & 2.67 & 14.51 & 1.73 & 12.26 & 0.68 \\
\hline Liver & 8.68 & 0.86 & 14. 05 & 3. 34 & 17.08 & 1.24 & 16.72 & 3.78 \\
\hline Bone & 2.83 & 0.84 & 2.87 & 0.51 & 2. 30 & 1.62 & 2.77 & 0.65 \\
\hline
\end{tabular}

\section{B}

\begin{tabular}{|c|c|c|c|c|c|c|c|c|}
\hline \multirow[t]{2}{*}[{}^{18}\mathrm{F}]{15} & \multicolumn{2}{|c|}{$5 \mathrm{~min}$} & \multicolumn{2}{|c|}{$15 \mathrm{~min}$} & \multicolumn{2}{|c|}{$30 \mathrm{~min}$} & \multicolumn{2}{|c|}{$60 \mathrm{~min}$} \\
\hline & Mean & $\mathrm{SD}$ & Mean & $\mathrm{SD}$ & Mean & $\mathrm{SD}$ & Mean & SD \\
\hline Brain & 3.52 & 0.53 & 4.71 & 0.68 & 5.26 & 0.77 & 5.02 & 0.22 \\
\hline Blood & 2.75 & 0.45 & 2.55 & 0.31 & 2.43 & 0.42 & 2.12 & 0.33 \\
\hline Muscle & 5.27 & 0.87 & 4. 39 & 0.50 & 3.79 & 0.90 & 2.95 & 0.49 \\
\hline Spleen & 4.71 & 1. 06 & 6.53 & 0.93 & 5.29 & 1.81 & 4.12 & 0.55 \\
\hline Heart & 19.99 & 4.00 & 13.85 & 2.30 & 9.62 & 1.67 & 7. 18 & 0.37 \\
\hline Lung & 8.66 & 1.23 & 7.48 & 0.69 & 5.61 & 0.97 & 4. 12 & 0.34 \\
\hline Pancreas & 8.21 & 1.09 & 7.24 & 0.81 & 5.01 & 1.11 & 3.79 & 0.39 \\
\hline Stomach & 2.96 & 0.91 & 4. 74 & 0.67 & 4.45 & 1.70 & 6.87 & 1.41 \\
\hline Small Intestine & 8.05 & 2. 82 & 10.65 & 4.20 & 14. 47 & 5.25 & 14. 10 & 6. 18 \\
\hline Kidney & 19. 37 & 4. 41 & 15. 39 & 1.51 & 12. 44 & 1.80 & 10.00 & 1. 19 \\
\hline Liver & 25.75 & 3. 46 & 26. 42 & 1.29 & 21.13 & 2.00 & 19. 38 & 1.96 \\
\hline Bone & 4. 11 & 1. 14 & 2.94 & 1.43 & 2. 34 & 0.52 & 2.68 & 0.66 \\
\hline
\end{tabular}

\subsection{General information.}

Unless otherwise mentioned, all the chemicals employed in the syntheses were purchased from commercial vendors and used without further purification. ${ }^{1} \mathrm{H}$ NMR spectra were obtained at $300 \mathrm{MHz}$ on Bruker spectrometers at room temperature. ${ }^{13} \mathrm{C}$ NMR spectra were obtained at $75 \mathrm{MHz}$ and ${ }^{19} \mathrm{~F}$ NMR spectra were obtained at $282 \mathrm{MHz} .{ }^{1} \mathrm{H}$ NMR chemical shifts were determined relative to internal $\left(\mathrm{CH}_{3}\right)_{4} \mathrm{Si}$ (TMS) at $\delta 0.00 \mathrm{ppm} .{ }^{13} \mathrm{C}$ NMR chemical shifts were determined relative to the signal of the solvent: $\mathrm{CDCl}_{3} \delta 77.16 \mathrm{ppm}$ 
or $\mathrm{CD}_{3} \mathrm{OD} \delta 49.00 \mathrm{ppm} .{ }^{19} \mathrm{~F}$ NMR chemical shifts were determined relative to $\mathrm{CFCl}_{3}$ at $\delta$ $0.00 \mathrm{ppm}$. Chemical shifts $(\delta)$ are reported in ppm and coupling constants are reported in Hertz. The multiplicities are abbreviated as follows: $\mathrm{s}=$ singlet, $\mathrm{d}=$ doublet, $\mathrm{t}=$ triplet, $\mathrm{m}$ $=$ multiplet, $\mathrm{br}=$ broad signal, $\mathrm{dd}=$ doublet of doublets, and so forth. Mass spectra were obtained on a mass spectrometer. High-resolution mass data were recorded on a highresolution mass spectrometer in the EI or ESI mode.

\subsection{Synthetic procedures}

General synthetic scheme

Method A:<smiles></smiles>

Method B:

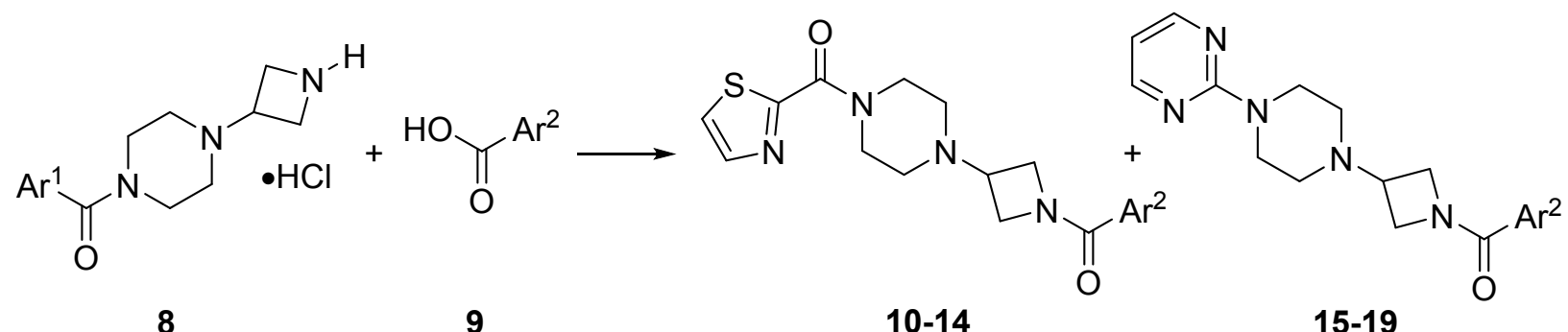

\subsection{HPLC traces of key compounds}

Compound 5, purity: 98.5\% 


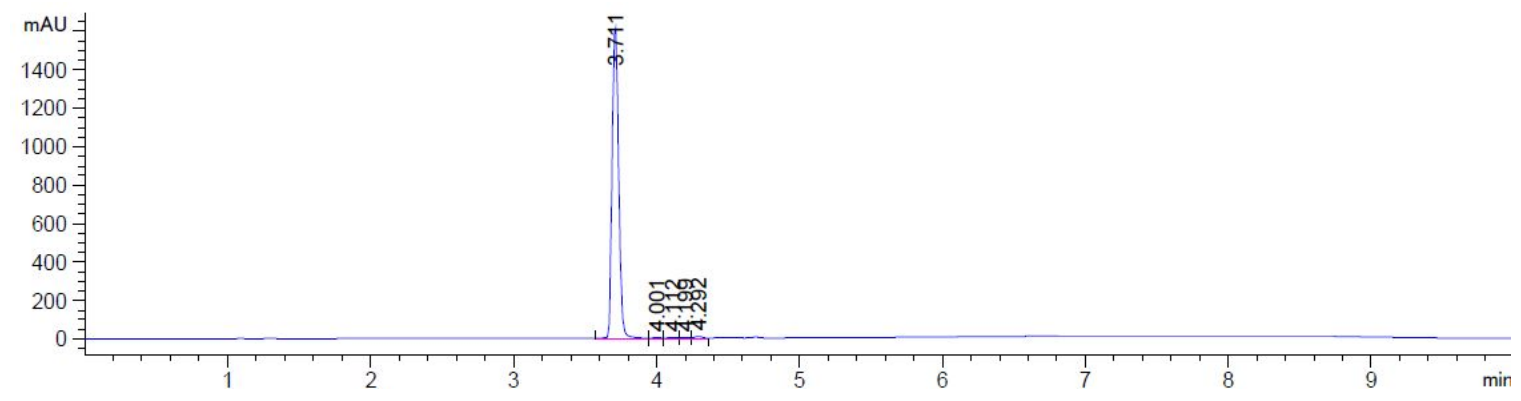

Compound 10, purity: 98.8\%

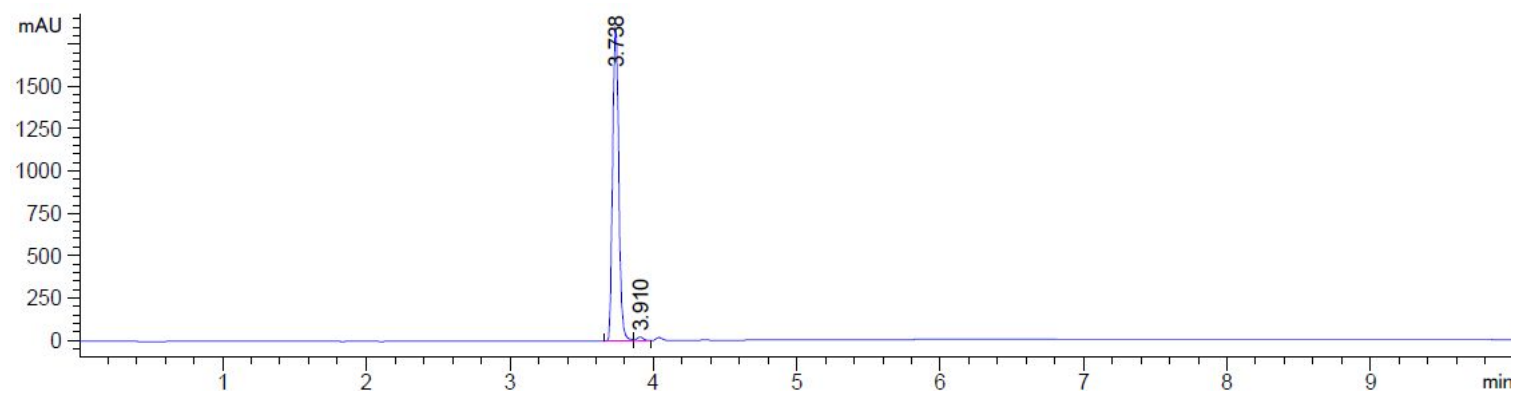

Compound 11, purity: 99.7\%

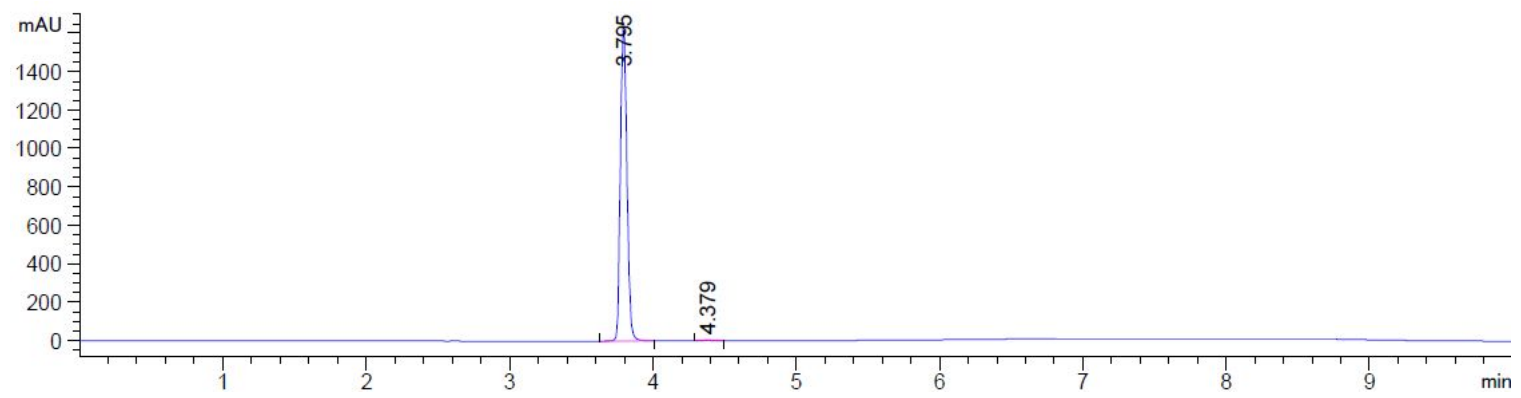

Compound 15, purity: 96.8\%

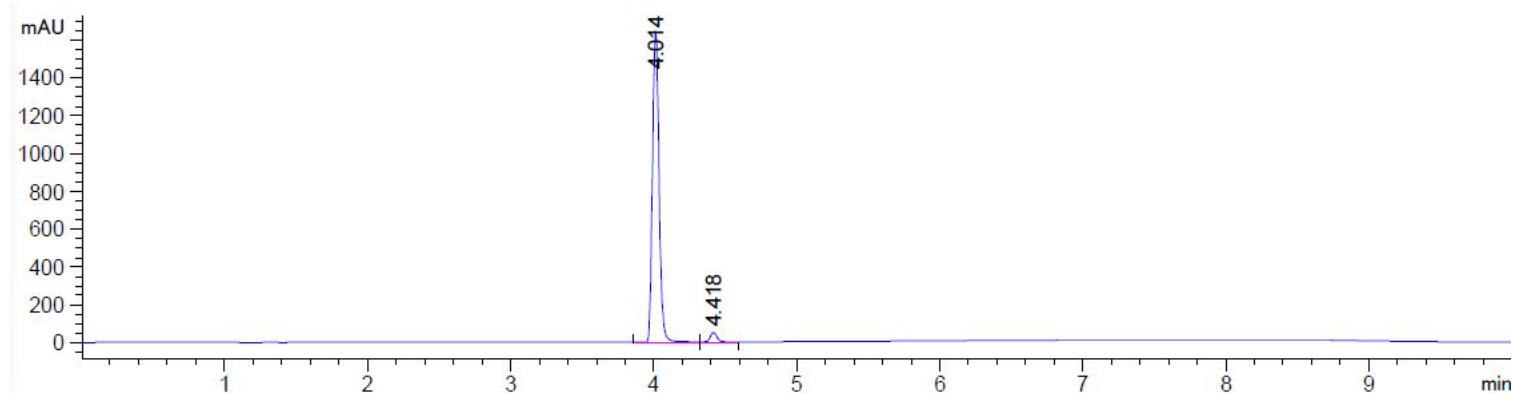

